\title{
Hydromagnetic Stagnation-Point Flow towards a Radially Stretching Convectively Heated Disk
}

\author{
S. Shateyi ${ }^{1}$ and O. D. Makinde ${ }^{2}$ \\ ${ }^{1}$ Department of Mathematics, University of Venda, Private Bag X5050, Thohoyandou 0950, South Africa \\ ${ }^{2}$ Institute for Advanced Research in Mathematical Modelling and Computations, Cape Peninsula University of Technology, \\ P.O. Box 1906, Bellville 7535, South Africa
}

Correspondence should be addressed to S. Shateyi; stanford.shateyi@univen.ac.za

Received 16 March 2013; Revised 23 May 2013; Accepted 26 May 2013

Academic Editor: Waqar Khan

Copyright ( 2013 S. Shateyi and O. D. Makinde. This is an open access article distributed under the Creative Commons Attribution License, which permits unrestricted use, distribution, and reproduction in any medium, provided the original work is properly cited.

\begin{abstract}
The steady stagnation-point flow and heat transfer of an electrically conducted incompressible viscous fluid are extended to the case where the disk surface is convectively heated and radially stretching. The fluid is subjected to an external uniform magnetic field perpendicular to the plane of the disk. The governing momentum and energy balance equations give rise to nonlinear boundary value problem. Using a spectral relaxation method with a Chebyshev spectral collocation method, the numerical solutions are obtained over the entire range of the physical parameters. Emphasis has been laid to study the effects of viscous dissipation and Joule heating on the thermal boundary layer. Pertinent results on the effects of various thermophysical parameters on the velocity and temperature fields as well as local skin friction and local Nusselt number are discussed in detail and shown graphically and/or in tabular form.
\end{abstract}

\section{Introduction}

The study of boundary layer flow over stretching sheet has been a subject of great interest due to its applications in designing cooling systems such as liquid metals, MHD generators, pumps, and flow meters. Many chemical engineering processes, such as metallurgical and polymer extrusion, involve cooling of molten liquids being stretched into cooling liquids (Abel and Mahesha [1], Bataller [2], and Abdou [3], among others). Various researchers have explored the flow over stretching surfaces in different circumstances. Munawar et al. [4] presented an analytic solution of flow of viscous fluid between two stretching disks with slip boundaries. Hayat et al. [5] investigated flow and heat transfer in second grade fluid over a stretching sheet subjected to convective boundary conditions. Shateyi and Motsa [6] numerically analysed variable viscosity on magnetohydrodynamic fluid flow and heat transfer over an unsteady stretching surface with Hall effect. Makinde and Sibanda [7] investigated the effects of chemical reaction on boundary layer flow past a vertical stretching surface in the presence of internal heat generation. Recently, Ashraf and Batool [8] carried out a numerical study of an axisymmetric steady laminar incompressible flow from an electrically conducted micropolar fluid over a stretching disk.

Stagnation-point flow appears in virtually all fields of science and engineering. A flow can be stagnated by a solid wall or a free stagnation point or a line can exist in the interior of the fluid domain. The study of stagnationpoint flow was pioneered by Hiemenz in 1911 [9] who solved the two-dimensional stagnation-point problem using a similarity transformation. Motsa et al. [10] formulated the Maxwell fluid for two-dimensional stagnation flow towards a shrinking sheet and then calculated the numerical solution of transformed non linear ordinary differential equations via the successive linearisation method. Bhattacharyya et al. [11] analyzed the effects of partial slip on steady boundary layer stagnation-point flow of an incompressible fluid and heat transfer towards a shrinking sheet. This investigation explored the conditions of the nonexistence, existence, uniqueness, and duality of the solutions of selfsimilar equations numerically. Makinde and Charles [12] presented computational dynamics of hydromagnetic stagnation flow towards a stretching sheet. Nadeem et al. [13] 
investigated by using similarity transformations the unsteady MHD boundary layer flow of a micropolar fluid near the forward stagnation point of a two-dimensional plane surface. Makinde [14] examined the hydromagnetic mixed convection stagnation point flow towards a vertical plate embedded in a highly porous medium with radiation and internal heat generation. Devi et al. [15] studied radiation effect on steady laminar hydromagnetic flow of a viscous, Newtonian, and electrically conducted fluid past a porous rotating infinite disk taking Hall current into account. Mahapatra and Nandy [16] presented a mathematical analysis for the magnetohydrodynamic (MHD) axi symmetric stagnation-point flow and heat transfer over a shrinking sheet which shrinks axisymmetrically in its own plane.

The above cited papers give more insight into the prediction of skin-friction as well as heat/mass transfer near stagnation regions of bodies in high speed flows and also in the design of thrust bearing and radial diffusers, drag reduction, transportation cooling, and thermal recovery.

The presence of a transverse magnetic field in boundary layer flows over moving surfaces is a basic and important problem in magnetohydrodynamic (MHD). The MHD flow and heat transfer for a viscous fluid over a stretching sheet has enormous applications in many engineering problems such as plasma studies, petroleum industries, and geothermal energy extractions. Chamkha, and Ahmed [17] investigated the similarity solution for an unsteady MHD stagnation-point flow of a three-dimensional porous body with heat and mass transfer. Motsa and Shateyi [18] obtained a numerical solution of MHD and rotating flow over a porous shrinking sheet by the spectral homotopy analysis method. Makinde and Aziz [19] explored the effects of the magnetic field on the viscous flow and heat transfer over a flat plate under a convective boundary condition. Butt and Ali [20] investigated the effects of magnetic field on the entropy generation during fluid flow and heat transfer due to radially stretching surface. Makinde et al. [21] analyzed the combined effects of buoyancy force, convective heating, Brownian motion, thermophoresis, and magnetic field on stagnation-point flow and heat transfer due to nanofluid flow towards a stretching sheet. Ibrahim et al. [22] analyzed the effect of magnetic field on stagnation-point flow and heat transfer due to nanofluid towards a stretching sheet.

Governing equations modelling MHD flow and heat transfer over stretching surfaces are highly nonlinear. To that end, exact solutions are impossible to obtain. Therefore, numerical solutions have always been developed, modified, and highbred, as a bid of getting more accurate and stable solutions. The current study seeks to extend the steady stagnation-point flow and heat transfer of an electrically conducted incompressible viscous fluid to the case where the disk surface is convectively heated and radially stretching. We propose to numerically solve the present problem using a recently developed iterative method known as spectral relaxation method (SRM); see Motsa et al. [23-25]. The SRM approach is based on transforming nonlinear ordinary differential equation into an iterative scheme. The iterative scheme is then blended with Chebyshev spectral method.

\section{Model Formulation}

Consider a steady stagnation-point flow of an electrically conducted incompressible viscous fluid towards a radially stretching convectively heated disk in the presence of a transverse magnetic field of strength $B_{0}$ applied parallel to the $z$-axis, as shown in Figure 1. The sheet is stretched with a linear velocity $u=U_{w}(r)=a r$, where $a$ is a real number. The bottom surface of the plate is assumed to be heated by convection from a hot fluid at temperature $T$, which provides a heat transfer coefficient, $h$. Based on this assumption, the boundary condition for the thermal field can be written as [26-28]. The induced magnetic field due to the motion of the electrically conducted fluid is negligible. It is also assumed that the external electrical field is zero and that the electric field due to the polarization of charges is negligible.

The relevant governing equations of fluid flow and heat transfer are

$$
\begin{gathered}
\frac{\partial}{\partial r}(r u)+\frac{\partial}{\partial z}(r w)=0, \\
u \frac{\partial u}{\partial r}+w \frac{\partial u}{\partial z}=U_{\infty} \frac{d U_{\infty}}{d r}+\nu \frac{\partial^{2} u}{\partial z^{2}}-\frac{\sigma B_{0}^{2}\left(u-U_{\infty}\right)}{\rho}, \\
u \frac{\partial T}{\partial r}+w \frac{\partial T}{\partial z}=\frac{k}{\rho c_{p}} \frac{\partial^{2} T}{\partial z^{2}}+\frac{\mu}{\rho c_{p}}\left(\frac{\partial u}{\partial z}\right)^{2}+\frac{\sigma B_{0}^{2}\left(u-U_{\infty}\right)^{2}}{\rho},
\end{gathered}
$$

with

$$
\begin{gathered}
u=U_{w}=a r, \quad w=0, \\
-k \frac{\partial T}{\partial z}=h\left(T_{f}-T\right) \quad \text { at } z=0, \\
u \longrightarrow U_{\infty}=b r, \quad T \longrightarrow T_{\infty} \quad \text { as } z \longrightarrow \infty .
\end{gathered}
$$

2.1. Similarity Transformation. To make the problem amenable, following Butt and Ali [20], among others, we introduce the following nondimensional quantities:

$$
\begin{aligned}
& \eta=\sqrt{\frac{b}{v}} z, \quad u(r, z)=b r f^{\prime}(\eta), \\
& w(r, z)=-2 \sqrt{b v} f(\eta), \quad \theta(\eta)=\frac{T-T_{\infty}}{T_{w}-T_{\infty}}, \\
& \nu=\frac{\mu}{\rho}, \quad \lambda=\frac{a}{b}, \quad \mathrm{Bi}=\frac{h}{k} \sqrt{\frac{b}{v}}, \quad \mathrm{Ha}=\frac{\sigma B_{0}^{2}}{\rho b}, \\
& \mathrm{Ec}=\frac{U_{\infty}^{2}}{c_{p}\left(T-T_{\infty}\right)}, \quad \operatorname{Pr}=\frac{\rho \nu c_{p}}{k} .
\end{aligned}
$$

Substituting (3) into (1) and (2), we obtain

$$
\begin{gathered}
f^{\prime \prime \prime}+2 f f^{\prime \prime}-f^{\prime 2}-\operatorname{Ha}\left(f^{\prime}-1\right)=-1, \\
\theta^{\prime \prime}+2 \operatorname{Pr} f \theta^{\prime}+\operatorname{PrEc} f^{\prime \prime 2}+\operatorname{PrEcHa}\left(f^{\prime}-1\right)^{2}=0 .
\end{gathered}
$$




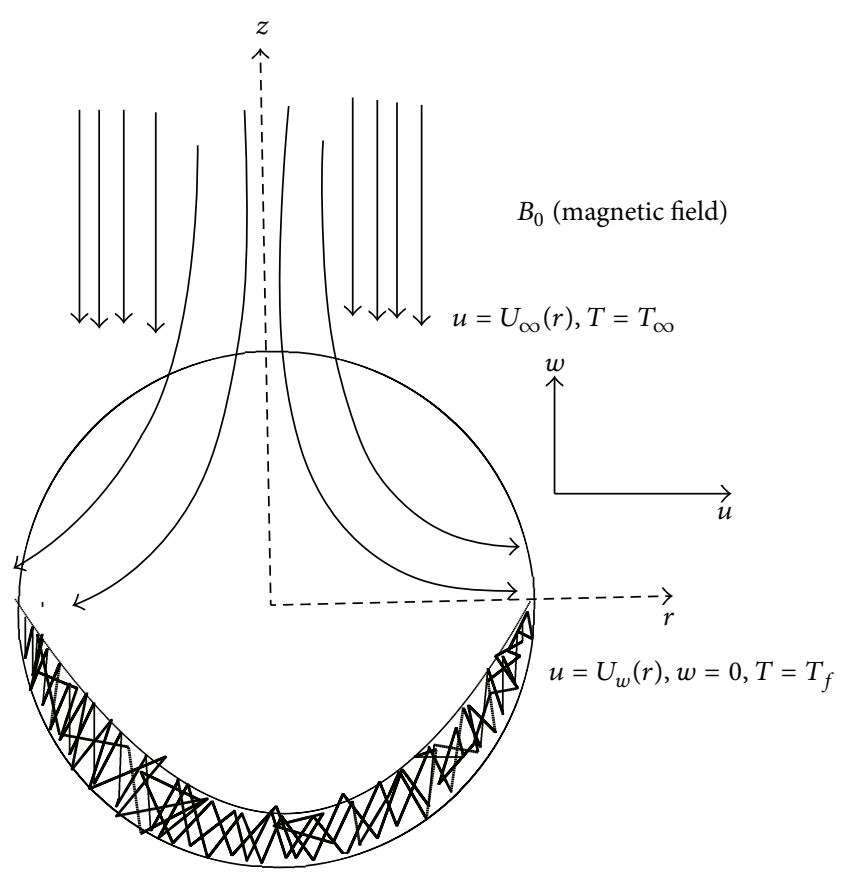

FIGURE 1: The physical model and the coordinate system for the problem.

With

$$
\begin{gathered}
f^{\prime}(0)=\lambda, \quad f(0)=0, \\
\theta^{\prime}(0)=\operatorname{Bi}(\theta(0)-1) \quad f^{\prime}(\infty)=1, \quad \theta(\infty)=0 .
\end{gathered}
$$

In the above equations, prime denotes differentiation with respect to $\eta$. The physical quantities of practical significance in this work are the local skin friction coefficient $C_{f}$ and the local Nusselt number $\mathrm{Nu}$, which are expressed as

$$
C_{f}=\frac{\tau_{w}}{\rho U_{\infty}^{2}}, \quad \mathrm{Nu}=\frac{r q_{w}}{k\left(T_{f}-T_{\infty}\right)},
$$

where $C_{f}$ is the skin friction, $\tau$ is the surface shear stress, and $q_{w}$ is the heat flux from the plate which are given by

$$
\tau_{w}=\left.\mu \frac{\partial u}{\partial z}\right|_{z=0}, \quad q_{w}=-\left.k \frac{\partial T}{\partial z}\right|_{z=0} .
$$

Substituting (8) into (7), we obtain

$$
\operatorname{Re}_{r}^{1 / 2} C_{f}=f^{\prime \prime}(0), \quad \operatorname{Re}_{r}^{-1 / 2} \mathrm{Nu}=-\theta^{\prime}(0),
$$

where $\operatorname{Re}_{r}=U_{\infty} r / v$ is the stagnation flow Reynolds number.

\section{Method of Solution}

The set of (4) and (5) together with the boundary conditions (6) are coupled nonlinear which are to be solved numerically using a spectral relaxation method (SRM). This method involves transforming (4) and (5) into a set of linear ordinary differential equations. The entire computation procedure is implemented using a program written in MATLAB computer language. From the process of numerical computation, the fluid velocity, temperature, the local skin friction coefficient, and the local Nusselt number are determined. The SRM algorithm starts with the assumption of having a system of $m$ nonlinear ordinary differential equations in $m$ unknowns functions $z_{i}(\eta), i=1,2, \ldots, m$ where $\eta \in[a, b]$ is the independent variable. To solve the resultant iterative scheme, we then use the Chebyshev pseudospectral method. The details of the spectral methods can be found in (Canuto et al. [29] and Trefethen [30]). Before applying the spectral method, the domain on which the governing equation is defined to the interval $[-1,1]$ on which the spectral method can be implemented. We use the transformation $\eta=(b-a)(\tau+1) / 2$ to map the interval $[a, b]$ to $[-1,1]$.

Now to apply the SRM on (4) together with (5), we set $f^{\prime}(\eta)=g(\eta)$ and then write the equations as the following set of equations:

$$
\begin{gathered}
f^{\prime}=g, \\
g^{\prime \prime}+2 f g^{\prime}-g^{2}-\mathrm{Ha} g+\mathrm{Ha}=-1, \\
\frac{1}{\mathrm{Pr}} \theta^{\prime \prime}+2 f \theta^{\prime}+\mathrm{Ec} g^{\prime 2}+\mathrm{EcHa}(g-1)^{2}=0,
\end{gathered}
$$

and the boundary conditions become

$$
\begin{array}{cl}
f(0)=0, & g(0)=\lambda, \quad \theta^{\prime}(0)=\operatorname{Bi}(\theta(0)-1), \\
g(\infty)=1, & \theta(\infty)=0 .
\end{array}
$$

In view of the SRM, we obtain the following iteration scheme:

$$
\begin{gathered}
f_{r+1}^{\prime}=g_{r}, \quad f_{r+1}(0)=0, \\
g_{r+1}^{\prime \prime}+2 f_{r+1} g_{r+1}^{\prime}-\mathrm{Hag}_{r+1}=-1+g_{r}^{2}-\mathrm{Ha}, \\
g_{r+1}(0)=\lambda, \quad g_{r+1}(\infty)=1, \\
\frac{1}{P r} \theta_{r+1}^{\prime \prime}+2 f_{r+1} \theta_{r+1}^{\prime}=-E c g_{r+1}^{\prime 2}-E c H a(g-1)^{2}=0, \\
\theta_{r+1}(0)=\frac{B i}{1+B i}, \quad \theta_{r+1}(\infty)=0 .
\end{gathered}
$$

We note that the equations now form a system of linear decoupled equations which can be solved iteratively for $r=1,2, \ldots$, starting from initial guesses/approximations $\left(g_{0}(\eta), \theta(\eta)\right)$.

Applying the Chebyshev pseudospectral method on (12) to (14) we obtain

$$
\begin{gathered}
A_{1} \mathbf{f}_{r+1}=B_{1}, \quad f_{r+1}\left(\tau_{\bar{N}}\right)=0, \\
A_{2} \mathbf{g}_{r+1}=B_{2}, \quad g_{r+1}\left(\tau_{\bar{N}}\right)=\lambda, \quad g_{r+1}\left(\tau_{0}\right)=1, \\
A_{3} \theta_{r+1}=B_{3}, \quad \theta_{r+1}\left(\tau_{\bar{N}}\right)=\frac{B i}{1+B i}, \quad \theta_{r+1}\left(\tau_{0}\right)=0,
\end{gathered}
$$


where

$$
\begin{gathered}
A_{1}=\mathbf{D}, \quad B_{1}=\mathbf{g}_{\mathbf{r}}, \\
A_{2}=\mathbf{D}^{2}+\operatorname{diag}\left[2 \mathbf{f}_{i+1}\right] \mathbf{D}-\mathrm{HaI}, \quad B_{2}=-1-\mathrm{Ha}+\mathbf{g}_{r}^{2}, \\
A_{3}=\frac{1}{\operatorname{Pr}} \mathbf{D}^{2}+\operatorname{diag}\left[2 \mathbf{f}_{i+1}\right] \mathbf{D}, \\
B_{3}=-\mathrm{Ec} g_{r+1}^{\prime 2}-\mathrm{EcHa}(g-1)^{2},
\end{gathered}
$$

where $I$ is the identity matrix of size $(\bar{N}+1) \times(\bar{N}+1)$, $\mathbf{f}, \mathbf{g}$, and $\theta$ are the values of $f, g$, and $\theta$, respectively, when evaluated at the grid points. Equations (13) to (15) constitute the SRM scheme. The initial approximation required to start the iterative process is

$$
\begin{gathered}
g_{0}(\eta)=\lambda-1+\eta+(1-\lambda) e^{-\eta}, \\
\theta_{0}(\eta)=\frac{\mathrm{Bi}}{1+\mathrm{Bi}} e^{-\eta},
\end{gathered}
$$

which are convenient random functions that satisfy the boundary conditions. The iteration is repeated until convergence is achieved. The convergence of the SRM scheme is defined in terms of the infinity norm as

$$
\mathrm{Er}=\operatorname{Max}\left(\left\|f_{r+1}-f_{r}\right\| ;\left\|\theta_{r+1}-\theta_{r}\right\|\right) .
$$

If the iteration scheme converges, the error Er will decrease with an increase in the number of iterations. Accuracy of the scheme was established by increasing the number of collocation points $N$ until the solutions are consistent and further increase does not change the value of the solutions.

\section{Results and Discussion}

The steady stagnation-point flow and heat transfer of an electrically conducted incompressible viscous fluid with the disk surface being convectively heated and radially stretching are numerically solved using the spectral relaxation method (SRM). We have used the bvp4c method to validate the SRM solution. The bvp4c is an in-built MATLAB solver for boundary value problems and is based on fourth-order Runge-Kutta schemes. The tolerance level for both methods was set to be $10^{-8}$. The figures were generated using $N=50$ and $\eta_{\infty}=15$. Figure 2 is used to illustrate how the SRM error reduces with increased number of iterations. The plot has been generated for different values of the magnetic parameter. A steep decrease of the error is being observed for all values of Ha. The decrease in the error is an indication that the method is convergent and gives stable solutions. The convergence rate is taken to be the number of iterations the solution takes to reach a specified accuracy level. For example, in the figure it shows that when $\mathrm{Ha}=4$, the solution will converge after fifteen iterations for the given accuracy level compared to eighteen when $\mathrm{Ha}=2$.

In order to gain physical insight into the flow properties, default numerical values of the parameters encountered in the problem are assigned. Their influences on the velocity and

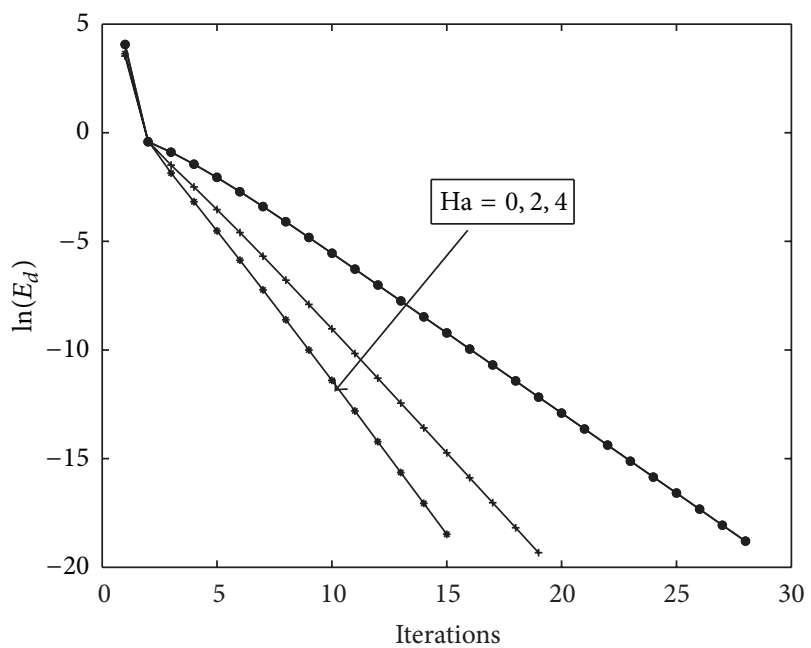

FIGURE 2: Influence of $\mathrm{Ha}$ on the velocity profiles when $\lambda=2, \operatorname{Pr}=$ $0.71, \mathrm{Bi}=0.3$, and $\mathrm{Ec}=0.2$.

TABLE 1: Values of the local Nusselt number for parameters Bi, Ec, $\lambda$, and $\mathrm{Ha}$.

\begin{tabular}{lcccc}
\hline $\mathrm{Bi}$ & Ec & $\lambda$ & Ha & $-\theta^{\prime}(0)$ \\
\hline 0.0 & & & & -0.07207353 \\
0.2 & 0.3 & 0.3 & 1 & 0.05773461 \\
0.5 & & & & 0.18754275 \\
2.0 & & & & 0.44715903 \\
\hline & 0.0 & & & 0.25961628 \\
0.3 & 0.2 & 0.3 & 1 & 0.18754275 \\
& 0.5 & & & 0.07994324 \\
\hline & & 0.0 & & 0.02032099 \\
0.3 & 0.3 & 0.2 & 1 & 0.11131746 \\
& & 0.5 & & 0.22034202 \\
\hline \multirow{3}{*}{0.3} & & & 0.0 & 0.33385485 \\
& 0.3 & 0.3 & 1.0 & 0.31735089 \\
& & & 5.0 & 0.26613250 \\
& & & 10.0 & 0.26613250 \\
\hline
\end{tabular}

temperature profiles as well as on the skin-friction and the Nusselt number are tabulated and/or displayed graphically.

Table 1 displays the effects of the Biot number (Bi), Eckert number $(\mathrm{Ec})$, and the velocity stretching parameter $(\lambda)$ on the Nusselt number $\left(-\theta^{\prime}(0)\right)$. From this table we observe that increasing the Biot number as expected causes the local Nusselt number to increase. Values of the Biot number more than 0.1 imply that the heat convection away from the surface is much faster than the heat inside the body. This gives rise to high temperature gradients at the surface. More heat is transferred from the wall when the values of the Biot number increase thus causing the Nusselt number to increase in our current study. We also observe from this table that as the Eckert number increases, the Nusselt number decreases. Physically, an increase in the Eckert number decreases the temperature gradient between the ambient and the plate. We observe that increasing the values of the Hartman numbers 
TABLE 2: Comparison of SRM solutions for $f^{\prime \prime}(0)$ against those of the bvp4c for $\lambda$ and $\mathrm{Ha}$ when $\operatorname{Pr}=0.71, \mathrm{Bi}=0.3$, and $\mathrm{Ec}=0.1$.

\begin{tabular}{lccc}
\hline & & $f^{\prime \prime}(0)$ & \\
$\lambda$ & Ha & SRM & bvp4c \\
\hline 0.0 & & 1.64532167 & 1.64532167 \\
0.2 & 1.0 & 1.38320821 & 1.38320821 \\
0.5 & & 0.92353421 & 1.38320821 \\
\hline & 0.0 & 0.78032335 & 0.78032335 \\
& 1.0 & 0.92353421 & 0.92353421 \\
0.5 & 5.0 & 1.35766817 & 1.35766817 \\
& 10.0 & 1.75767520 & 1.75767520 \\
\hline
\end{tabular}

leads to lowering the values of the Nusselt number. Application of a strong magnetic field reduces the flow velocity which in turn increases the temperature distributions within the fluid flow. This physically explains why heat transfer at the wall is reduced as Ha is increased. Similar observations were made by Butt and Ali [20]. Lastly, we clearly see from the table that the Nusselt number is increased when the values of the velocity parameter are increased.

Table 2 displays the influence of the Hartman number $\mathrm{Ha}$ and velocity parameter $\lambda$. The skin friction is greatly reduced by increasing the velocity parameter. As can be seen from the velocity boundary conditions, $\lambda$ is increased by increasing the stretching parameter $a$. Stretching the surface radially reduces the drag force on the wall surface thus reducing the skin friction. Stretching of the surface may lead to smothering of the surface area thereby reduces the drag force on the wall. We also observe in Table 2 that the Hartman number has significant effect on the skinfriction. As the magnetic strength increases, the dragging effect is clearly seen by the significant increments in the skin friction. The influence of different parameters on the velocity field $f^{\prime}(\eta)$ and temperature profile $\theta(\eta)$ is depicted in Figures 3 to 9 . In this table we observe an excellent agreement between the two methods for up to eight decimal places. However, the SRM converges much faster than the bvp4c both in terms of CPU time and number of iterations. This gives us much confidence in the SRM solutions.

Figure 3 depicts the effects of the Hartmann number $\mathrm{Ha}$ on the velocity distributions. In this figure, we observe that the velocity decreases with $\eta$ as the values of $\mathrm{Ha}$ are increased. Thus, the presence of the magnetic field reduces the momentum boundary layer thickness and increases the power needed to stretch the sheet. The presence of a moderate magnetic field can be used to stabilize the flow thereby delaying the transition from laminar to turbulent. Physically, the presence of a transverse magnetic field gives rise to a drag force known as Lorentz force which results in retarding the velocity field. Similar results were arrived at by Ibrahim et al. [22].

Figure 4 displays the effect of increasing the velocity parameter $\lambda$ on the velocity profiles. Increasing the stretching parameter causes the velocity to increase and reduces the boundary layer. The fluid flow is aided as the radially stretched surface is stretched. This explains why the velocity

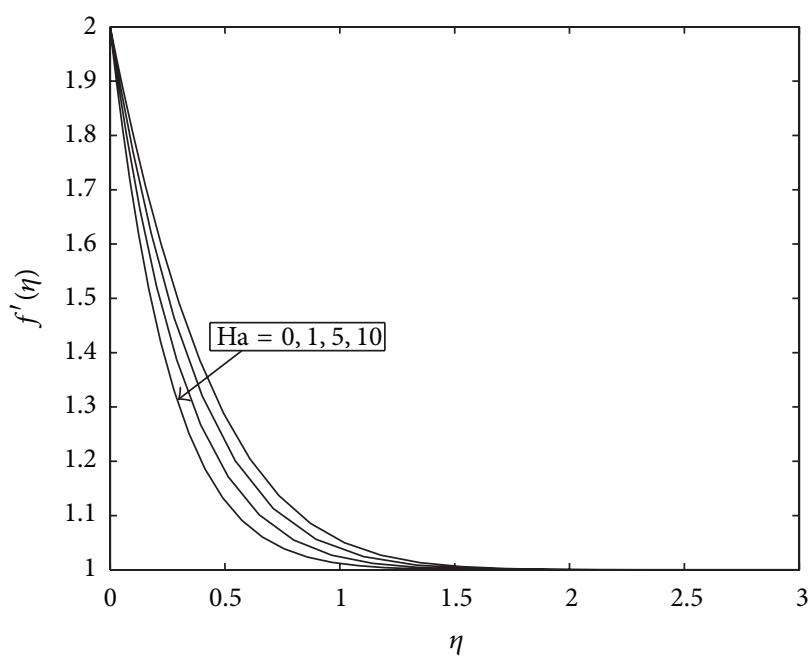

FIGURE 3: Influence of $\mathrm{Ha}$ on the velocity profiles when $\lambda=2, \mathrm{Pr}=$ $0.71, \mathrm{Bi}=0.3$, and $\mathrm{Ec}=0.2$.

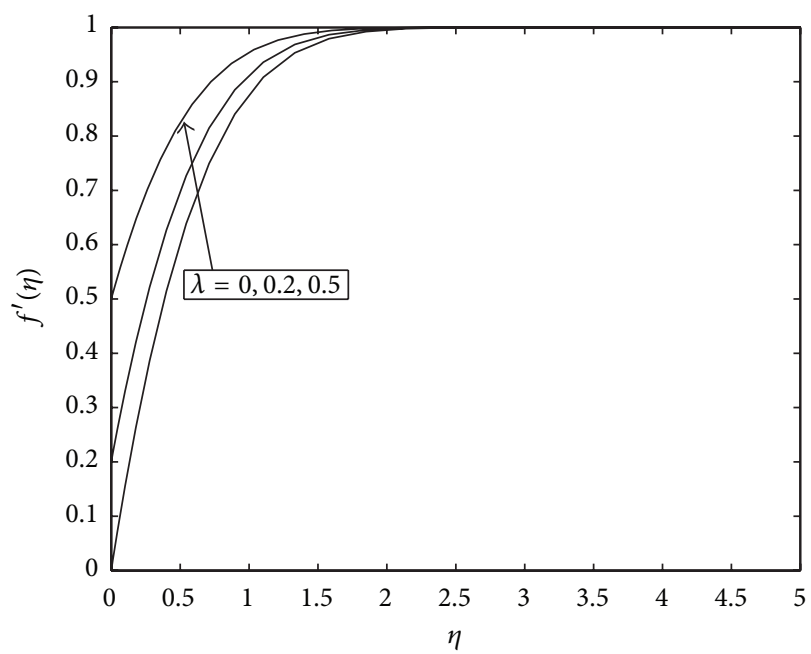

FIgURE 4: Variation of the velocity profiles with $\lambda$ when $\operatorname{Pr}=0.71$, $\mathrm{Ha}=1, \mathrm{Bi}=0.3$, and $\mathrm{Ec}=0.3$.

increases when the stretching parameter increases. The maximum value for the velocity is a unity (one) in dimensionless variables so as the stretching parameter increases, the velocity tends to converge to this value, thus explaining why the boundary layer thickness is reduced.

In Figure 5 we depict the effect of the Biot number on the temperature profiles. The temperature distributions within the fluid flow clearly increase when the Biot number increases. By the mathematical definition of the Biot number increasing its values means that the convective heat transfer coefficient increases thereby enhancing more heat transfer from the surface. This then causes the fluid to heat up thus increasing the fluid temperature distributions. The influence of the Eckert number Ec on the temperature profiles is shown on Figure 6. We observe that the temperature is an increasing function of Ec. This is because increasing the values of the Eckert number generates heat in the fluid due to frictional 


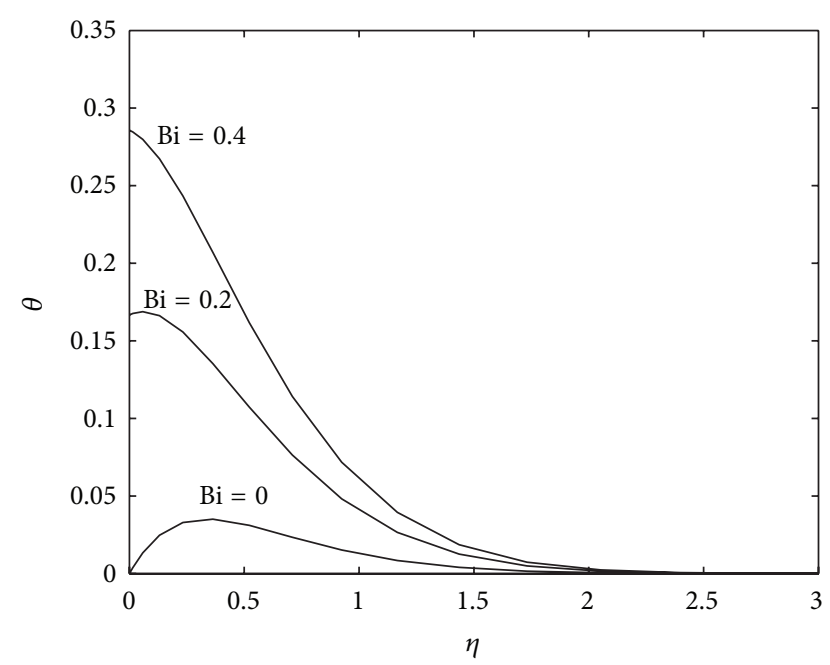

FIGURE 5: Influence of Biot number Bi on the temperature profiles when $\operatorname{Pr}=0.3, \mathrm{Ha}=1, \lambda=0.2$, and $\mathrm{Ec}=0.2$.

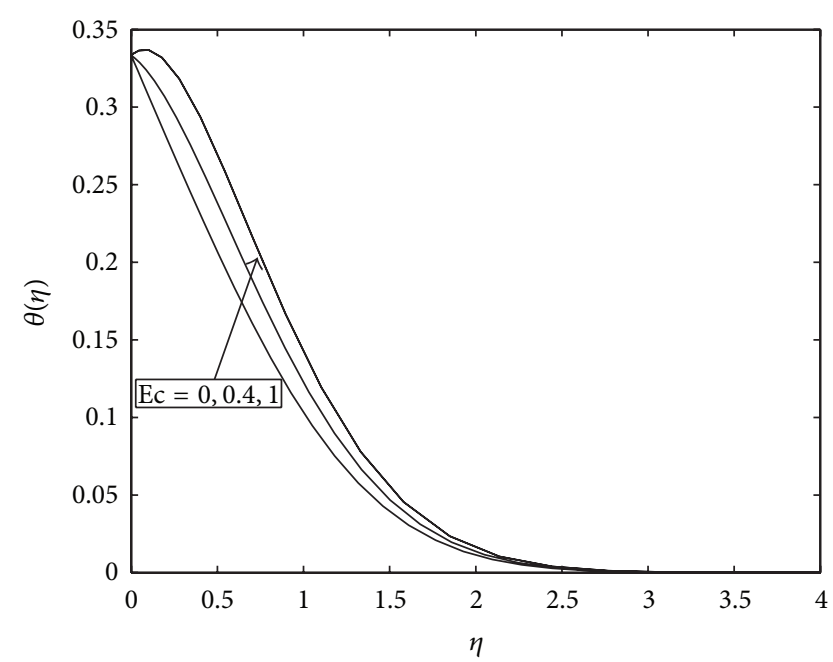

FIGURE 6: Influence of Eckert number Ec on the temperature profiles when $\operatorname{Pr}=0.3, \mathrm{Ha}=1, \lambda=0.2$, and $\mathrm{Bi}=0.3$.

heating. Thus the effect of increasing $\mathrm{Ec}$ is to enhance the temperature at any point.

The influence of the Hartmann on the fluid temperature is displayed in Figure 7. Increasing the magnetic field strength reduces the Nusselt number and hence increases the fluid temperature. Physically, the magnetic field has a stabilizing effect of the fluid velocity flow and thus increasing the temperature distribution within the fluid flow. Figure 8 displays the effect of increasing the velocity parameter $\lambda$ on the fluid temperature. The temperature is reduced as $\lambda$ increases. Lastly, Figure 9 depicts the effect of the Prandtl number on the temperature. It can clearly be observed that the temperature as well as the thermal boundary layer rapidly decreases with increasing values of the Prandtl number. Physically, an increase in the Prandtl number means an increase in fluid velocity which in turn causes a decrease in the temperature.

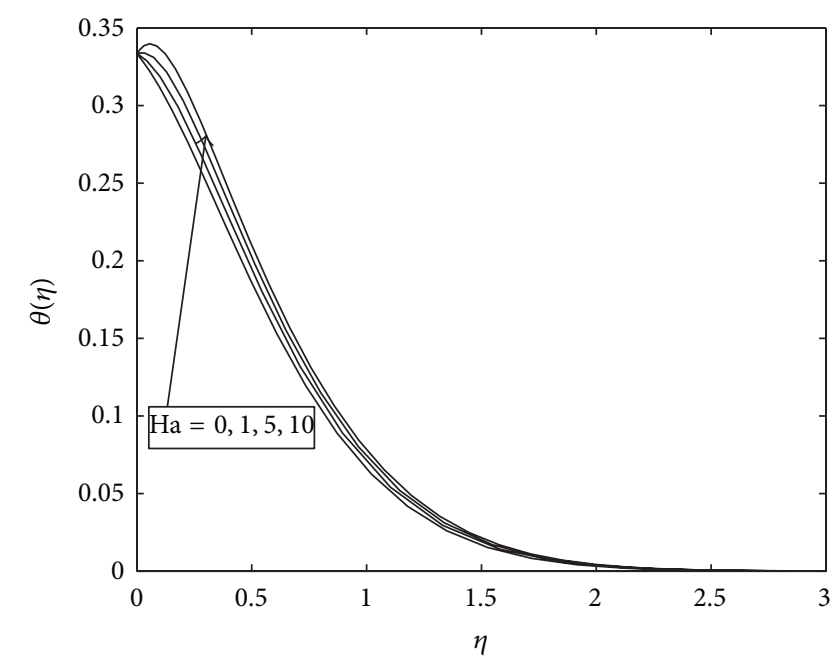

FIgURE 7: Influence of Hartmann number $\mathrm{Bi}$ on the temperature profiles when $\operatorname{Pr}=0.3, \mathrm{Bi}=0.3, \lambda=0.2$, and $\mathrm{Ec}=0.2$.

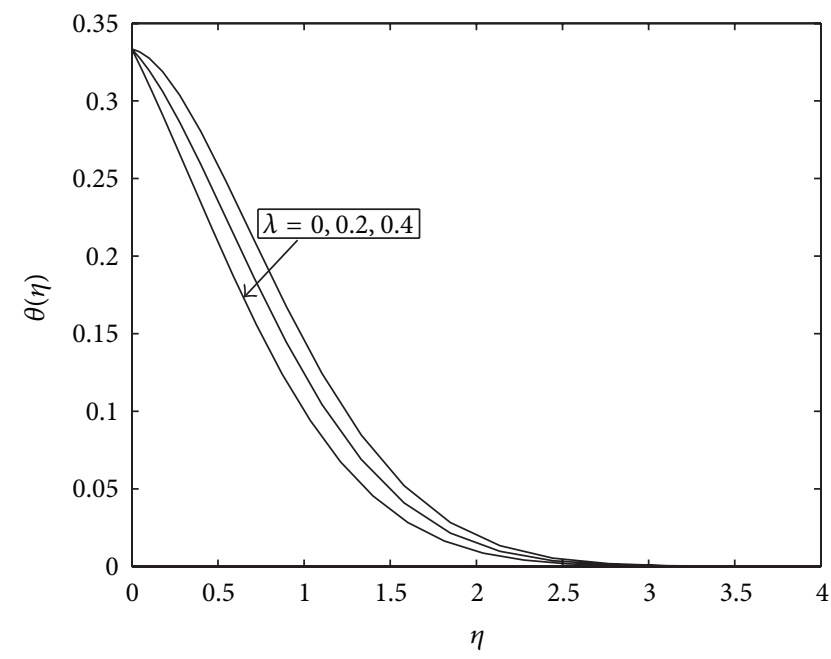

FIGURE 8: Influence of velocity parameter on the temperature profiles when $\mathrm{Pr}=0.3, \mathrm{Ha}=1, \mathrm{Bi}=0.3$, and $\mathrm{Ec}=0.2$.

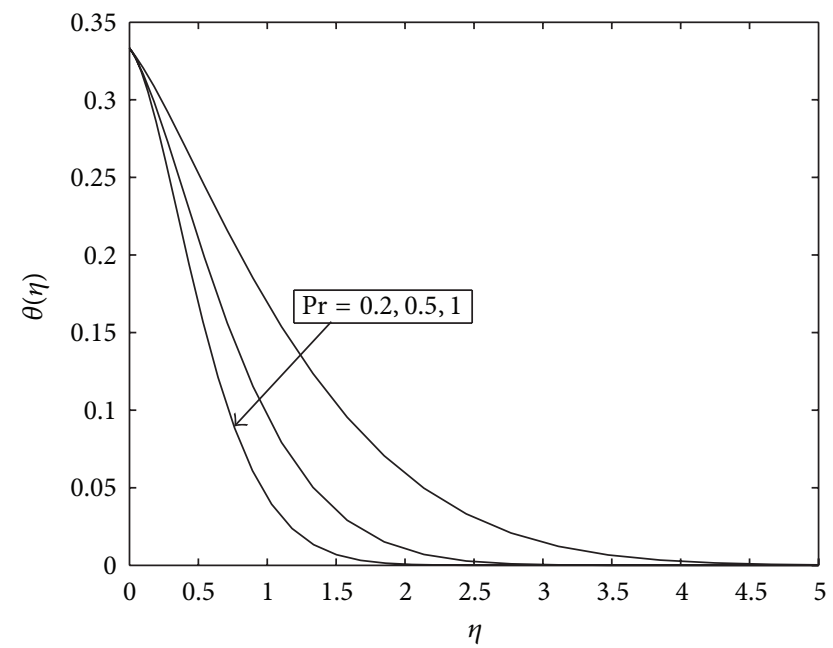

FIgURE 9: Variation of the temperature profiles with $\operatorname{Pr}$ when $\mathrm{Bi}=$ $0.3, \mathrm{Ha}=1, \lambda=0.2$, and $\mathrm{Ec}=0.2$. 


\section{Conclusion}

This work is a worthwhile attempt to study the effects of convective heating, viscous dissipation, and Joule heating on the stagnation-point flow and heat transfer of an electrically conducted fluid towards a radially stretching disk. An effective relaxation spectral algorithm with Chebyshev spectral scheme has been adopted to solve the resulting system of non linear differential equations subject to the convective boundary conditions. The accuracy of the SRM is validated against the MATLAB in-built bvp4c routine for solving boundary value problems. The combined effects of the convective heating and the magnetic interaction parameter are studied in detail. The velocity field was found to increase as the Harthmann number and velocity parameter increase. The fluid temperature increases by increasing values of Biot number, Eckert number, and the Hartmann number; it decreases as the Prandtl number and velocity parameter increase. These findings were also observed by Hayat et al. [5] in their investigations of the flow and heat transfer in a second grade fluid over a stretching sheet subjected to convective boundary conditions. We also found in this study that the rate of heat transfer at the surface increases as the Biot number (convective parameter) increases. A similar conclusion was arrived at by Abu Bakar et al. [26].

\section{Nomenclature}

$\begin{array}{ll}\mathrm{a}: & \text { Initial stretching rate } \\ \mathrm{b}: & \text { Stagnation-point flow rate } \\ \mathrm{Bi}: & \text { Biot number } \\ B_{0}: & \text { Magnetic field of constant strength } \\ \mathrm{C}_{f}: & \text { Skin friction coefficient } \\ c_{p}: & \text { Heat capacity at constant pressure } \\ \mathrm{Ec}: & \text { Eckert number } \\ \mathrm{h}: & \text { Heat coefficient at the disk surface } \\ \mathrm{Ha}: & \text { Hartmann number } \\ \mathrm{k}: & \text { Thermal conductivity coefficient } \\ \mathrm{Nu}: & \text { Local Nusselt number } \\ \mathrm{Pr}: & \text { Prandtl number } \\ q_{w}: & \text { Heat flux from the plate } \\ \mathrm{Re}_{r}: & \text { Stagnation flow Reynolds number } \\ (\mathrm{r}, \mathrm{z}): & \text { Polar coordinates } \\ (\mathrm{u}, \mathrm{w}): & \text { Velocity components } \\ U_{\infty}: & \text { Free stream velocity } \\ \mathrm{T}: & \text { Temperature } \\ T_{f}: & \text { The convective fluid temperature below } \\ & \text { the moving sheet } \\ T_{\infty}: & \text { Free stream temperature. } \\ & \end{array}$

\section{Greek Letters}
$\rho$ : Fluid density
$\sigma$ : Electrical conductivity
$\eta$ : Similarity variable
$\lambda$ : Velocity ratio parameter
$\tau$ : Surface shear stress
$\mu$ : Coefficient of viscosity
$v$ : Kinematic viscosity
$\theta$ : Dimensionless temperature.

\section{Acknowledgments}

The authors wish to acknowledge financial support from the University of Venda and NRF.

\section{References}

[1] M. S. Abel and N. Mahesha, "Heat transfer in MHD viscoelastic fluid flow over a stretching sheet with variable thermal conductivity, non-uniform heat source and radiation," Applied Mathematical Modelling, vol. 32, no. 10, pp. 1965-1983, 2008.

[2] R. C. Bataller, "Viscoelastic fluid flow and heat transfer over a stretching sheet under the effects of a non-uniform heat source, viscous dissipation and thermal radiation," International Journal of Heat and Mass Transfer, vol. 50, no. 15-16, pp. 31523162, 2007.

[3] M. M. M. Abdou, "Effect of radiation with temperature dependent viscosity and thermal conductivity on unsteady a stretching sheet through porous media," Nonlinear Analysis, vol. 15, no. 3, pp. 257-270, 2010.

[4] S. Munawar, A. Mehmood, and A. Ali, "Effects of slip on flow between stretchable disks using optical homotopy analysis method," Canadian Journal of Pure and Applied Sciences, vol. 1, no. 2, pp. 50-67, 2011.

[5] T. Hayat, S. A. Shehzad, M. Qasim, and S. Obaidat, "Flow of a second grade fluid with convective boundary conditions," Thermal Science, vol. 15, no. 2, pp. S253-S261, 2011.

[6] S. Shateyi and S. S. Motsa, "Variable viscosity on magnetohydrodynamic fluid flow and heat transfer over an unsteady stretching surface with Hall effect," Boundary Value Problems, vol. 2010, Article ID 257568, 20 pages, 2010.

[7] O. D. Makinde and P. Sibanda, "Effects of chemical reaction on boundary layer flow past a vertical stretching surface in the presence of internal heat generation," International Journal of Numerical Methods for Heat and Fluid Flow, vol. 21, no. 6, pp. 779-792, 2011.

[8] M. Ashraf and K. Batool, "MHD flow and heat transfer of a micropolar fluid over a stretchable disk," Journal of Theoretcal and Applied Mechanics, vol. 51, no. 1, pp. 25-38, 2013.

[9] K. Hiemenz, "Die Grenzschicht in Einem in Dem Gleichformingen Flussigkeitsstrom Eingetauchten Gerade Kreiszlinder," Dingler Polytech Journal, vol. 326, pp. 321-410, 1911.

[10] S. S. Motsa, Y. Khan, and S. Shateyi, "A new numerical solution of Maxwell fluid over a shrinking sheet in the region of a stagnation point," Mathematical Problems in Engineering, vol. 2012, Article ID 290615, 11 pages, 2012.

[11] K. Bhattacharyya, S. Mukhopadhyay, and G. C. Layek, "Slip effects on boundary layer stagnation-point flow and heat transfer towards a shrinking sheet," International Journal of Heat and Mass Transfer, vol. 54, no. 1-3, pp. 308-313, 2011.

[12] O. D. Makinde and W. M. Charles, "Computational dynamics of hydromagnetic stagnation flow towards a stretching sheet," Applied and Computational Mathematics, vol. 9, no. 2, pp. 243251, 2010.

[13] S. Nadeem, M. Hussain, and M. Naz, "MHD stagnation flow of a micropolar fluid through a porous medium," Meccanica, vol. 45 , no. 6, pp. 869-880, 2010.

[14] O. D. Makinde, "Heat and mass transfer by MHD mixed convection stagnation point flow toward a vertical plate embedded in a highly porous medium with radiation and internal heat generation," Meccanica, vol. 47, no. 5, pp. 1173-1184, 2012. 
[15] S. P. Devi, D. Anjali, and R. Uma, "Effects of thermal radiation on hydromagnetic flow dueto a porous rotating disk with hall effect," Journal of Applied Fluid Mechanics, vol. 5, no. 2, pp. 1-8, 2012.

[16] T. R. Mahapatra and S. K. Nandy, "Momentum and heat transfer in MHD axisymmetric stagnation-point flow over a shrinking sheet," Journal of Applied Fluid Mechanics, vol. 6, no. 1, pp. 121129, 2013.

[17] A. J. Chamkha and S. E. Ahmed, "Similarity solution for unsteady MHD flow near a stagnation point of a threedimensional porous body with heat and mass transfer, heat generation/absorption and chemical reaction," Journal of Applied Fluid Mechanics, vol. 4, no. 3, pp. 87-94, 2011.

[18] S. S. Motsa and S. Shateyi, "A new approach for the solution of three-dimensional magnetohydrodynamic rotating flow over a shrinking sheet," Mathematical Problems in Engineering, vol. 2010, Article ID 586340, 15 pages, 2010.

[19] O. D. Makinde and A. Aziz, "Boundary layer flow of a nanofluid past a stretching sheet with a convective boundary condition," International Journal of Thermal Sciences, vol. 50, no. 7, pp. 13261332, 2011.

[20] A. S. Butt and A. Ali, "Effects of magnetic field on entropy generation in flow and heat transfer to a radially stretching surface," Chinese Physics Letters, vol. 30, no. 2, pp. 1-5, 2013.

[21] O. D. Makinde, W. A. Khan, and Z. H. Khan, "Buoyancy effects on MHD stagnation point flowand heat transfer of ananofluidpast aconvectively heated stretching/shrinking sheet," International Journal of Heat and Mass Transfer, vol. 62, pp. 526-533, 2013.

[22] W. Ibrahim, B. Shankar, and M. M. Nandeppanavar, "MHD stagnation point flow and heat transfer due to nanofluid towards a stretching sheet," International Journal of Heat and Mass Transfer, vol. 56, pp. 1-9, 2013.

[23] S. S. Motsa, P. G. Dlamini, and M. Khumalo, "Solving hyperchaotic systems using the spectral relaxation method," Abstract and Applied Analysis, vol. 2012, Article ID 203461, 18 pages, 2012.

[24] S. S. Motsa, P. Dlamini, and M. Khumalo, "A new multistage spectral relaxation method for solving chaotic initial value systems," Nonlinear Dynamics, vol. 72, no. 1-2, pp. 265-283, 2013.

[25] S. S. Motsa and Z. G. Makukula, "On spectral relaxation method approach for steady von Krmn flow of a Reiner-Rivlin fluid with Joule heating, viscous dissipation and suction/injection," Central European Journal of Physics, vol. 11, no. 3, pp. 363-374, 2013.

[26] N. A. Abu Bakar, W. M. K. A. W. Zaimi, R. Abdul Hamid, B. Bidin, and A. Ishak, "Boundary lyer flow over a stretching sheet with a convective boundary condition and slip effect," World Applied Sciences Journal, vol. 17, pp. 49-53, 2012.

[27] A. Aziz, "A similarity solution for laminar thermal boundary layer over a flat plate with a convective surface boundary condition," Communications in Nonlinear Science and Numerical Simulation, vol. 14, no. 4, pp. 1064-1068, 2009.

[28] A. Ishak, "Similarity solutions for flow and heat transfer over a permeable surface with convective boundary condition," Applied Mathematics and Computation, vol. 217, no. 2, pp. 837842, 2010.

[29] C. Canuto, M. Y. Hussaini, A. Quarteroni, and T. A. Zang, Spectral Methods in Fluid Dynamics, Springer, Berlin, Germany, 1988.

[30] L. N. Trefethen, Spectral Methods in MATLAB, SIAM, Philadelphia, Pa, USA, 2000. 


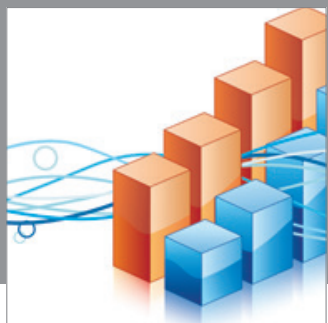

Advances in

Operations Research

mansans

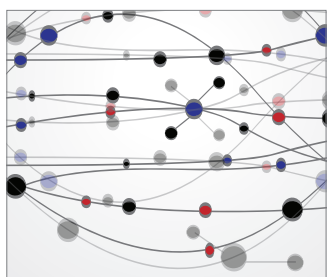

The Scientific World Journal
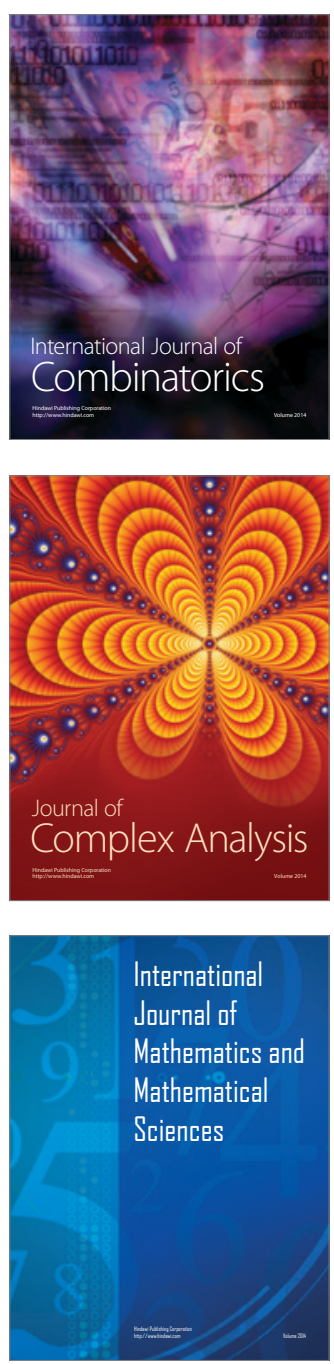
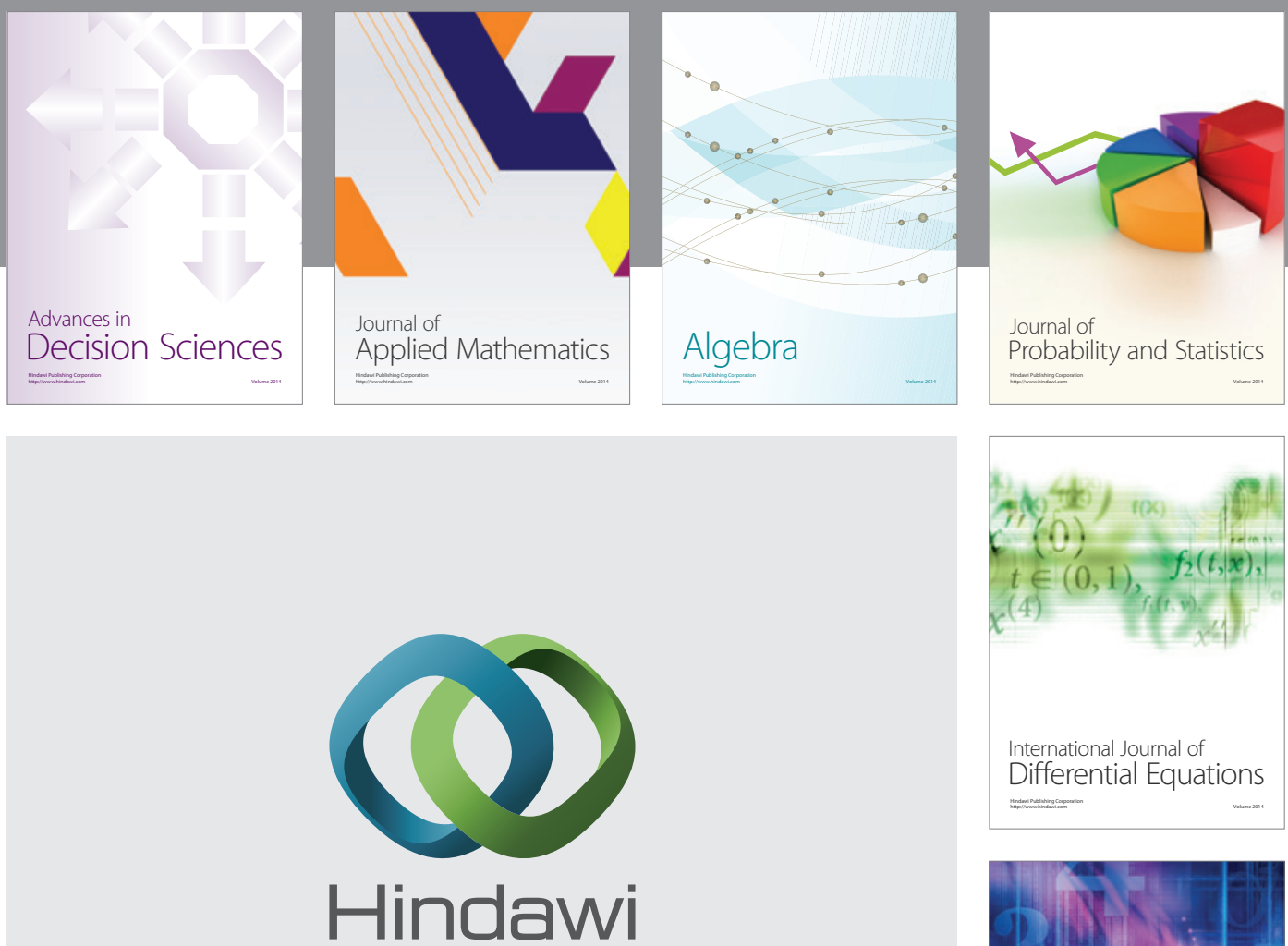

Submit your manuscripts at http://www.hindawi.com
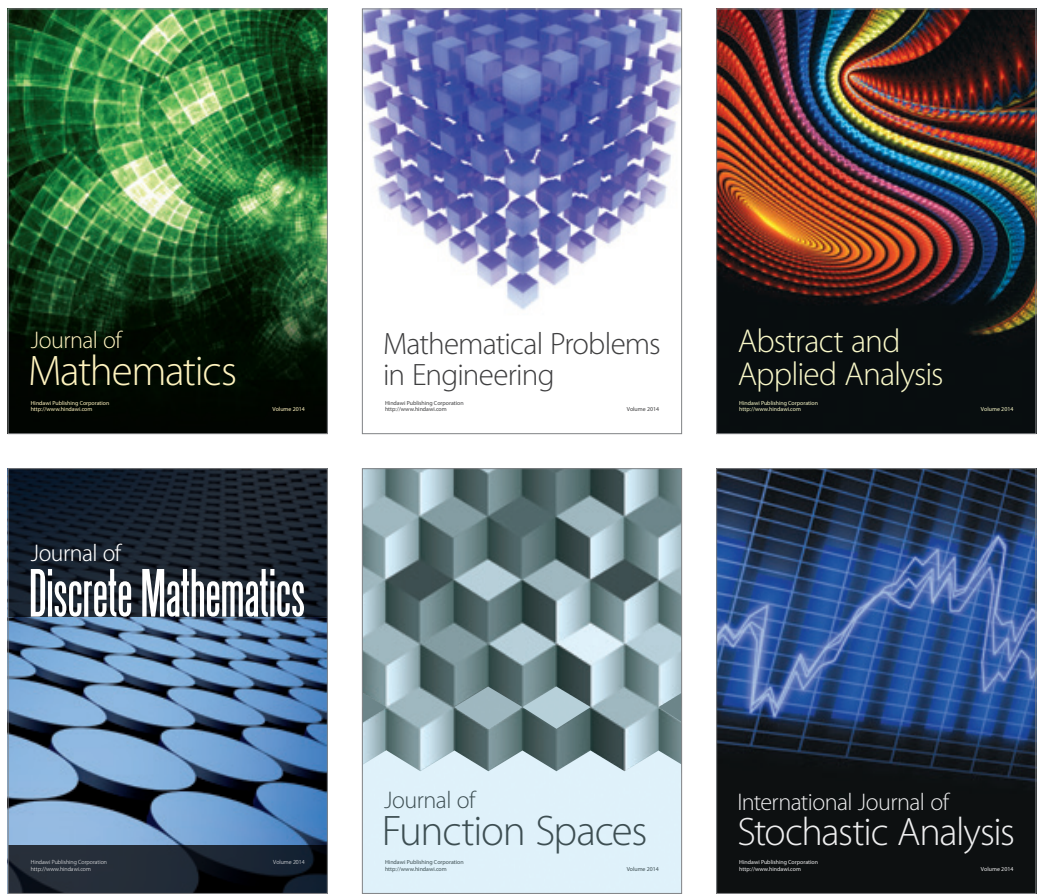

Journal of

Function Spaces

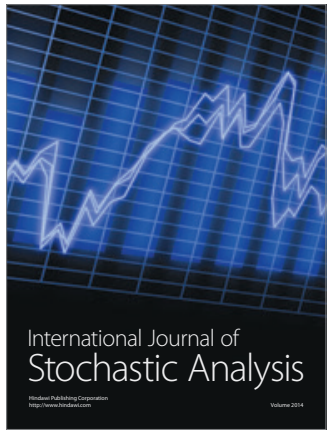

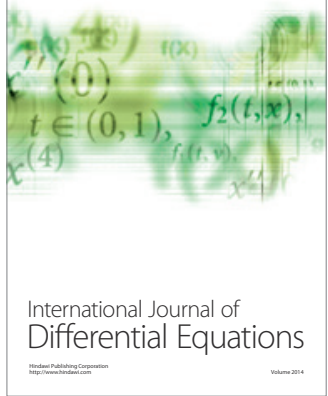
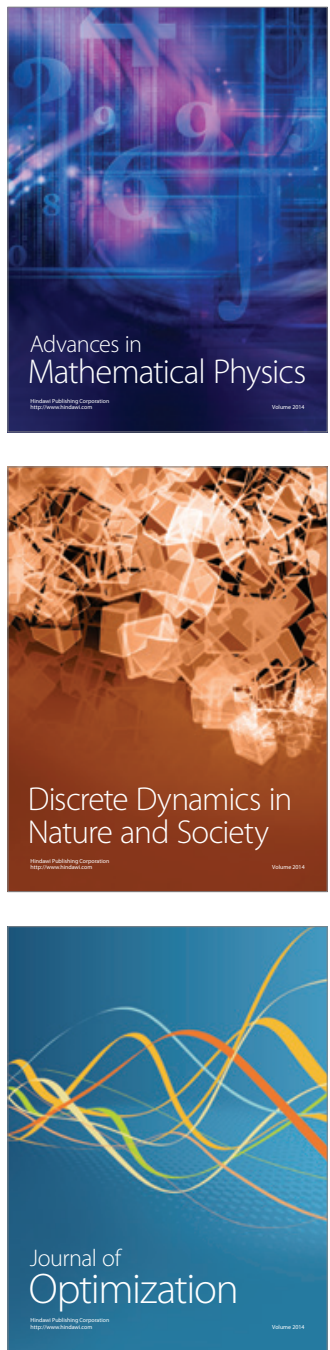